\title{
Re-thinking the role of the dorsal striatum in egocentric/response strategy
}

\author{
Fanny Botreau ${ }^{1}$ and Pascale Gisquet-Verrier ${ }^{1,2 *}$ \\ Centre de Neurosciences Paris-Sud, UMR 8195, Centre National de la Recherche Scientifique, Orsay, France \\ 2 UMR 8195, Université Paris-Sud, Orsay, France
}

Edited by:

Susan J. Sara,

Université Pierre et Marie Curie,

France; Collège de France, France

\section{Reviewed by:}

Martine Ammassari-Teule, Consiglio Nazionale delle Ricerche, Italy

Francoise Schenk,

Université de Lausanne, Switzerland

*Correspondence:

Pascale Gisquet-Verrier, Centre de

Neurosciences Paris-Sud, UMR 8195

Université Paris-Sud, Bât. 446, 91405

Orsay Cedex, France.

e-mail: pascale.gisquet@u-psud.fr
Rats trained in a dual-solution cross-maze task, which can be solved by place and response strategies, predominantly used a response strategy after extensive training. This paper examines the involvement of the medial and lateral dorsal striatum (mDS and IDS) in the choice of these strategies after partial and extensive training. Our results show that rats with IDS and mDS lesions used mainly a response strategy from the early phase of training. We replicated these unexpected data in rats with IDS lesions and confirmed their tendency to use the response strategy in a modified cross-maze task. When trained in a dual-solution water-maze task, however, control and lesioned rats consistently used a place strategy, demonstrating that IDS and $\mathrm{mDS}$ lesioned rats can use a place strategy and that the shift towards a response strategy did not systematically result from extensive training. The present data did not show any clear dissociation between the mDS and IDS in dual solution tasks. They further indicate that the dorsal striatum seems to determine the strategies adopted in a particular context but cannot be considered as a neural support for the response memory system. Accordingly, the role of the lateral and medial part of the dorsal striatum in egocentric/response memory should be reconsidered.

Keywords: memory system, behavioural strategy, dorsal striatum, egocentric, response/place strategies, excitotoxic lesions, cross-maze task, rat

\section{INTRODUCTION}

The idea that one can learn about a particular experience in more than one way is compatible with the idea that memory is supported by parallel, and largely independent neural circuits which operate in an interactive manner to optimize behavioural performance (McDonald and White, 1993; Eichenbaum, 2001). Among the various forms of memory, the declarative, cognitive or spatial memory system is described as being independent of the procedural or cue or response memory system (McDonald and White, 1994; Devan and White, 1999; Devan et al., 1999) and is sub-served by different brain systems, the hippocampus and the dorsal striatum, respectively (Potegal, 1972; Mishkin and Petri, 1984; Packard et al., 1989; McDonald and White, 1993).

Several studies have shown impairment in the acquisition of tasks that require the use of an egocentric strategy after lesions of the dorsal striatum (Potegal, 1969; Cook and Kesner, 1988; Kesner et al., 1993; DeCoteau and Kesner, 2000). This position has, however, been challenged by other studies postulating that dorsal striatum is not involved in egocentric discriminations (Divac et al., 1967, 1978; Kirkby, 1969; Pisa and Cyr, 1990; Oliveira et al., 1997). One reason for these contradictory results may be the heterogeneous nature of the dorsal striatum which is divided mainly into two distinct subregions.

The medial dorsal striatum ( $\mathrm{mDS}$, or associative striatum, the counterpart of the caudate nucleus in primates) receives sensory information and is innervated by the medial prefrontal and cingulate cortices (Divac and Diemer, 1980; Sesack et al., 1989). Lesions of the mDScan produce cognitive learning deficits fairly similar to those observed after hippocampal system damage in some learning tasks (Divac, 1968; Kolb, 1977; Whishaw et al., 1987; Dunnett, 1990; Kesner et al., 1996; Devan et al., 1999), suggesting that it may be considered as a part of the spatial memory system (Yin and Knowlton, 2004). Alternatively, large-scale loop circuits that include both medial striatal subregions and medial prefrontal cortical areas have been implicated in strategy shifting in maze learning tasks (Palencia and Ragozzino, 2004; Tzavos et al., 2004; McCool et al., 2008), indicating that mDS may be important in the competition between memory systems in the present task.

In the rodent, the lateral part of the dorsal striatum (the sensorimotor striatum, equivalent to the putamen in primates) receives input from the dorsolateral frontal and parietal cortices (Wise and Jones, 1977; Donoghue and Herkenham, 1986). Lesions of the dorsolateral striatum (IDS) disrupt sensory orientation and motor control (Barth et al., 1990; Pisa and Cyr, 1990; Castro-Alamancos and Borrel, 1995). There is evidence that IDS is involved in the acquisition of a win-stay strategy (Packard et al., 1989; McDonald and White, 1993) and in the formation of stimulus-response associations (Devan et al., 1999), suggesting that IDS is a part of the response memory system.

The cross-maze task is a standard dual-solution task which is used to assess the respective contributions of the response and place learning systems and to further determine their relative involvement during the course of learning. Initially introduced by Tolman (Tolman et al., 1946, 1947), this task requires to consistently reaching, the same goal arm of a T-maze from the same start arm, to 
get reward. Two different strategies are possible to master this task: rats may either adopt a particular body turn at the choice point, using an egocentric response strategy, or reach a particular spatial location associated with the reinforcer, using a place strategy. Rats can also solve the task by using a third strategy, i.e. the association between discrete stimuli and the reinforcer, irrespective of any relationship with spatial cues and explicit behaviour. This cue strategy which seems to depend on the integrity of the same system as the response strategy, can easily be confounded with either the place or response strategies, depending upon the circumstances. Initial studies indicate that rats are more likely to adopt a place strategy early in training and a response strategy later (Restle, 1957), suggesting that hippocampal processes control expression of learning in early training trials and that striatal processes take over later in training (Packard and McGaugh, 1996).

Curiously, experiments having investigated the effects of dorsostriatal pre-training lesions in the dual-solution task performed with a cross-maze are very few. In an early study, it was shown that rats with dorso-striatal lesions tended to exhibit more place than response strategies (Thompson et al., 1980). Later, lidocaineinduced inactivation of the dorsal hippocampus and dorsal striatum were shown to decrease the expression of place and response solutions, respectively (Packard and McGaugh, 1996), suggesting that both main memory systems compete for control over behaviour. This result has, however, been challenged by others indicating that dorso-striatal lesions have no influence on the initial acquisition of the task, nor on the strategy used during an early probe test (Oliveira et al., 1997). More recently, Yin and Knowlton (2004) investigating the respective role of the lateral and the medial part of the dorsal striatum in a cross-maze task, reported an enhanced tendency to use the response strategy for rats with $\mathrm{mDS}$ lesions and place strategy for rats with IDS lesions, although the lack of environmental cues rendered the place strategy difficult to adopt. The present study sought to clarify the specific contributions of the lateral and medial parts of the dorsal striatum in the initial choice of strategy and in the shift from a place to a response strategy, with extended training.

In an attempt to separate different possibilities we addressed three specific issues. First, we investigated the dissociation between response and place strategies in a classical cross-maze situation in control rats and in rats with IDS or mDS lesions. Second, we confirmed the unexpected results obtained in the first experiment and further tested the validity of the probe test to determine the favoured strategy used by the rats. Finally, control rats and rats with lesions to the IDS and mDS were tested in a spatial task performed in a water maze, using a similar dual-solution procedure, in order to control the processing of the spatial information and to investigate whether the shift from a place to a response strategy with extended training can be generalized to other training situations.

\section{MATERIALS AND METHODS \\ ANIMALS}

Male Sprague-Dawley rats (250-300 g; Iffa-Credo, Lyon, France) were housed in pairs in wire-mesh cages and maintained on a 12-h light-dark cycle with the lights on between 7 a.m. and 7 p.m. in a temperature-controlled colony room $\left(21^{\circ} \mathrm{C}\right)$. All rats were regularly handled before the beginning of behavioural testing in order to familiarize them with experimenters. Before training, rats were reduced to $85 \%$ of their ad lib feeding weights over 7 days and this deprivation was maintained throughout the experiment. All experimental procedures were carried out in accordance with the European Communities Council Directive (24 XI.1986) and within the guidelines of CNRS and the French Agricultural and Forestry Ministry (decree 87848; licence number, A91429).

\section{SURGERY}

Rats underwent surgery 1 week after their arrival and were randomly assigned to the different surgical conditions. Rats were anaesthetized with pentobarbital (50 mg/kg/i.p.) and mounted in a stereotaxic frame (Narishige Instrument, Tokyo, Model SR6) on a thermal blanket to maintain their body temperature $\left(37-38^{\circ} \mathrm{C}\right)$. Holes were drilled in the skull. Microinjections were made through glass micropipettes (external tip diameter: $70-80 \mu \mathrm{m}$, Clark Electromedical Instruments, Pangbourne, England) glued to the needle of a $10-\mu$ Hamilton syringe which was filled with liquid paraffin solution (paraffin oil, Merck, Strasbourg, France).

The mDS and IDS lesions were induced with an N-methyl Daspartate (NMDA) agonist. mDS: $0.2 \mu \mathrm{l}$ of NMDA (Sigma Chemical Company, St Louis, MO, USA; concentration: $252 \mathrm{mM}$, dissolved in phosphate buffered saline; $\mathrm{pH}$ 7.4; Van Golf Racht-Delatour and El Massioui, 1999) were injected 0.2 and $1.6 \mathrm{~mm}$ anterior to bregma; 1.8 and $1.6 \mathrm{~mm}$ lateral to midline, and 4.4 and $3.8 \mathrm{~mm}$ below the dura; IDS: $0.2 \mu$ l of NMDA were injected 0.2 and $0.6 \mathrm{~mm}$ anterior to bregma; 3.8 and $3.2 \mathrm{~mm}$ lateral to midline, and 4.4 and $3.8 \mathrm{~mm}$ below the dura. Neurotoxic solutions were injected at a rate of $0.1 \mu \mathrm{l} / \mathrm{min}$ and the micropipette was left in place for a further $5 \mathrm{~min}$. At the end of the surgery, the incision was sutured. For Sham-operated rats, no penetration with the glass micropipette was made, in order to prevent any lesion in these rats. Behavioural training began 10-15 days after surgery.

Experiment 1 involved 80 rats: 17 Sham mDS, 18 Sham lDS, 21 mDS and 24 lDS rats. Experiment 2 involved 22 lDS rats.

\section{HISTOLOGY}

After completion of the behavioural testing, rats were overdosed with pentobarbital $(120 \mathrm{mg} / \mathrm{kg})$ and perfused transcardially with saline, followed by a $4 \%$ buffered formalin solution. Brains were post fixed in buffered formalin and cryoprotected by immersion in three successive sucrose solutions (12, 16 and 18\%) for $48-72 \mathrm{~h}$. The brains were sectioned $(40 \mu \mathrm{m})$ on a freezing microtome and every third section was taken and stained with NeuN antibody (mouse anti-neuronal nuclei, monoclonal antibody; Sigma Chemical Company, St Louis, MO, USA). Sections were subsequently observed under microscope and lesion extents were digitized and redrawn using Photoshop software (Adobe, San Jose, USA), according to a method developed by B. Delatour (Delatour and Gisquet-Verrier, 1996).

\section{BEHAVIOURAL TASKS}

Cross-maze task

Cross-maze apparatus. The apparatus was an elevated $(47 \mathrm{~cm})$, wooden eight-arm radial maze painted in grey, with arms $(80 \mathrm{~cm} \times 12 \mathrm{~cm}$ and plastic walls $2-\mathrm{cm}$ high) radiating from a central platform $(30 \mathrm{~cm}$ in diameter). The apparatus was placed on a 
rotating device and located in a testing room $(265 \mathrm{~cm} \times 265 \mathrm{~cm})$ that contained several extra-maze cues. To prevent entries in the non used arms, five plexiglass blocks $(12 \mathrm{~cm} \times 15 \mathrm{~cm} \times 1 \mathrm{~cm})$ were placed at the entrance of these arms, giving the maze a T-shape with food-cups at the end of the west and east arms. The maze was located in a lit room containing extra-maze cue in moderate density, including a 40-W lamp placed in a corner, a radio playing in another corner, some objects, and a large picture on each of the walls. The position of the seated experimenter was fixed during each training trial. The experimenter stood in the maze room, close to the start arm (south or north).

Protocol of the cross-maze task (see Figure 3A). On the first 2 days, rats were placed in the start box of the cross-maze and allowed to explore the non-baited apparatus for $5 \mathrm{~min}$. On the third day, rats consumed 10 pieces of chocolate cereal (Kellogs) in a $25 \mathrm{~cm} \times 25 \mathrm{~cm} \times 35 \mathrm{~cm}$ plastic box. According to the time spent in each arm during the 2-day habituation, the goal arm was determined as the non-preferred arm (west or east).

Training began on the subsequent day. During each trial, rats were placed in the start arm facing away from the maze and trained to reach their non-preferred side in order to get a reinforcer placed in the cup at the end of the goal arm (four/five pieces of chocolate cereal; $50 \mathrm{mg}$ ). Indirect entries into the baited arm or failure to reach the baited food cup within $1 \mathrm{~min}$ were scored as an incorrect response. A correction procedure was used throughout training as rats reaching the non-baited arm were allowed to trace back to the baited goal arm. Rats were then returned to the holding cage, placed behind the start arm of the maze, for a 30-s intertrial interval. In order to minimize the use of intra-maze (odours) cues from the apparatus, the maze was turned $45-135^{\circ}$ clockwise relative to the experimenter between trials and plexiglass blocks were moved to different arms in order to reshape the maze in a cross-maze, before a new trial was begun. Each rat received four consecutive trials per day.

On day 8 , training was replaced by a single non-reinforced probe trial. Rats were placed in the opposite (North) arm to that of the usual start arm and the entrance to the south arm was blocked. Rats were allowed to make an entry into either the east or the west maze arms. Rats entering the same arm as that during training, therefore reaching the usually baited arm, were designated place learners. Rats entering the opposite arm, and thus making the same body turn response as during training were designated response learners. On day 9-15 of training, food-rewarded trials (four per day) were reinstated. On day 16, a second probe trial was given using the same procedure as that used for the first probe trial.

Protocol of the complementary cross-maze task (see Figure 4A). A modified version of the initial task during which the two starting points were used alternately, was conducted in some rats. From the usual start point, the task was identical to the initial task, but when rats started from the north point, half were required to use a place strategy (i.e. always reaching the same physical goal arm), while the other half were required to use a response strategy (i.e. always providing the same body turn). Rats received four consecutive trials per day, using different combinations of start arms. Rats were allowed to visit a single arm on each trial, except on the first training session, where a corrective procedure was used.

\section{Water-maze task}

Water-maze apparatus. The water maze was a circular whitepainted metal tank ( $1.50 \mathrm{~m}$ in diameter, $0.40 \mathrm{~m}$ in height $)$, raised $65 \mathrm{~cm}$ above the room floor. It was located in a room $(3.1 \mathrm{~m} \times 3.4 \mathrm{~m})$ containing several extra-maze cues and was filled to a depth of $27 \mathrm{~cm}$ with water $\left(25 \pm 1^{\circ} \mathrm{C}\right)$ made opaque with white non-toxic tempera paint (Brenntag, opacifier 631). The entire pool was virtually divided in four quadrants (North West, NW; North East, NE; South West, SW; South East, SE) of equal size by two diagonal lines running from the centre of the pool. During training, a circular white painted platform (PF, $10 \mathrm{~cm}$ in diameter, $26 \mathrm{~cm}$ in height) was submerged $1.5 \mathrm{~cm}$ below the surface of the water, except during the first training session when the platform was visible (dark, $1 \mathrm{~cm}$ above the surface of the water). Latencies and routes were recorded by a videotrack (Viewpoint 4.0, version 6.28) connected to a camera placed above the water maze. The experimenter stood in the adjacent room of the maze room. Extra-maze cues consisted of several visual cues fixed onto the walls.

Protocol of the water-maze task (see Figure 6A). On day 1-7, rats were placed into the water at the NW start position, facing the wall of the water maze. For half the rats, the platform was located in the NE quadrant while for the other half, the platform was located in the SW quadrant. The latencies to swim to the platform were recorded. Rats that failed to find the platform within $60 \mathrm{~s}$ were guided by hand, and a failed trial was recorded. Rats were allowed to remain on the platform for $30 \mathrm{~s}$; they were then dried during the 30-s intertrial interval. On day 1 of training, a black visible platform was used. From day 2 of training, a white platform placed $1.5 \mathrm{~cm}$ below the surface of the water was used. Each rat received four identical and consecutive trials per day.

On day 8 , a single $60 \mathrm{~s}$ probe trial was given at the end of the training session (i.e. after 32 training trials). The platform was removed from the pool and rats were placed in the start position opposite to that used during training. The time spent swimming within each quadrant was recorded. On day 9-14, rats were retrained in the usual training conditions with the platform. On day 15 , a probe trial, similar to that on day 8 , was given (i.e. after 60 training trials).

\section{STATISTICS}

Statistical analyses concerning the various parameters in the experiments were performed using analysis of variance (ANOVA) with lesion-type as between-subjects factors and session as within-subjects factor, using VAR3 software (Rouanet et al., 1990). Chi-square $\left(\chi^{2}\right)$ analyses were computed on the place and response strategies made by rats during the probe trials in the cross-maze and to determine potential changes in strategies between both probe trials.

\section{RESULTS \\ HISTOLOGY}

Reconstructions of mDS and IDS and lesions at different rostrocaudal levels are illustrated in Figure 1. To be included in the mDS lesioned group, lesions had to be limited to the medial part of the dorsal striatum associated with extension of the ventricles and with only minor encroachment on the globus pallidus or the cortex. To be included in the IDS lesion group, lesions had to be limited to the lateral part of the 


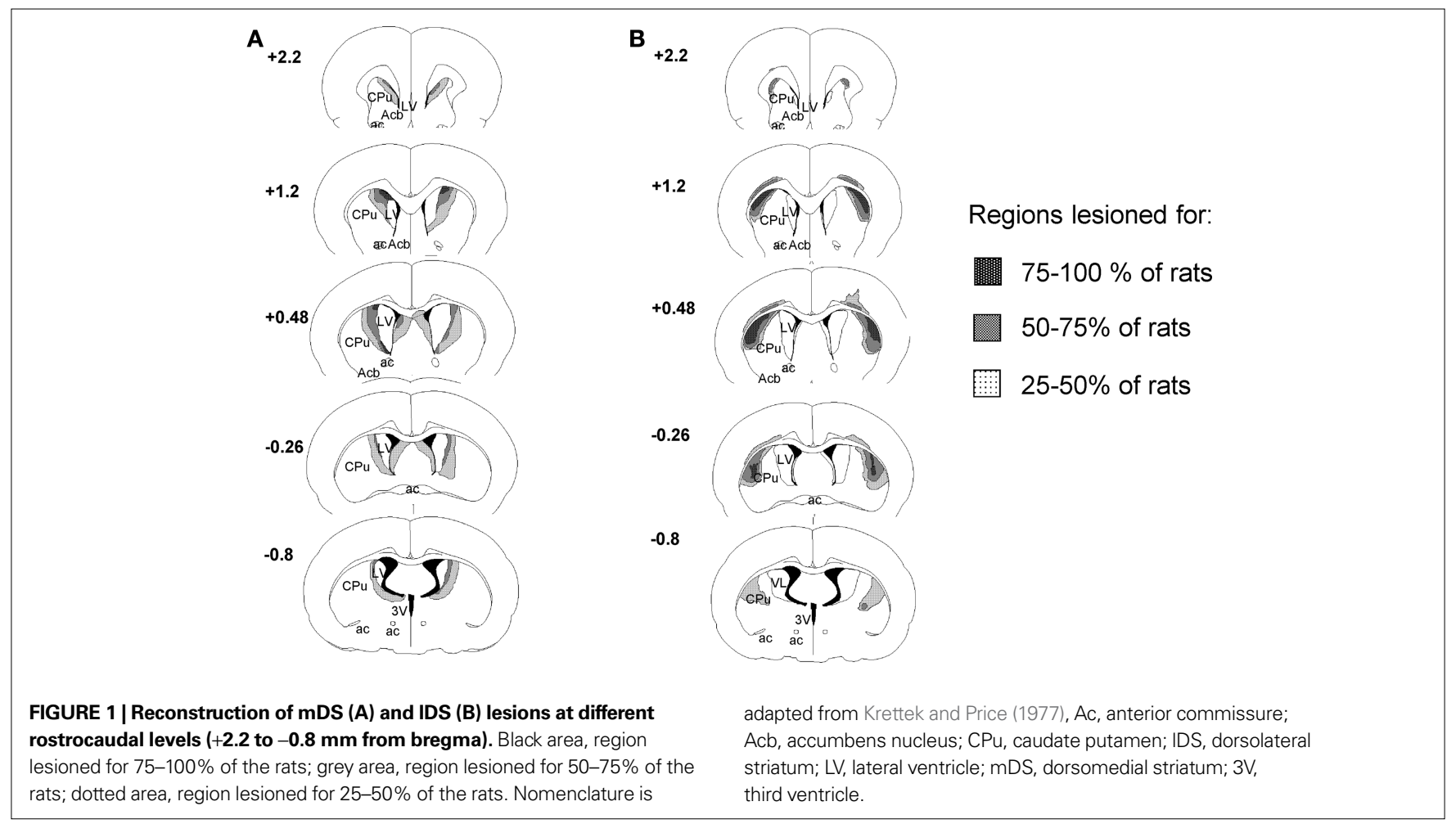

dorsal striatum with moderate extension of the ventricles and only minor encroachment on the globus pallidus or the cortex. Careful exams for adjacent and surrounding regions of the target site did not show any detectable cell loss due NMDA injections.

In Experiment 1, a total of 11 rats with lesions to IDS and 3 rats with lesions to $\mathrm{mDS}$ were discarded from behavioural analyses due to cortical encroachment (IDS: $n=7$; mDS: $n=2$ ), asymmetric lesions (IDS: $n=3$; mDS: $n=1$ ), or lesions that were too restricted (IDS: $n=1$ ).

In experiment 2, 18 rats have been included in IDS lesioned group for behavioural analyses. Others were discarded due to cortical encroachment $(n=3)$ or asymmetric lesions $(n=1)$.

\section{EXPERIMENT 1: CROSS-MAZE TASK}

As Sham mDS $(n=17)$ and Sham IDS $(n=18)$ rats behaved similarly in every aspects of acquisition and probe trials, a single combined control group was used for subsequent analyses. In all, the 84 rats were assigned to four groups: $\operatorname{Sham}(n=35), \operatorname{mDS}(n=18)$ and IDS $(n=13)$.

Sham-operated rats and rats with excitotoxic lesions of the medial and lateral part of the dorsal striatum were trained for 16 days (four trials a day) to reach a constant goal baited arm from a constant start arm (Figure 2).

\section{Motor activity during pre-training (see Figure 2C)}

During the pre-training days, rats in different lesion conditions exhibited differences in the numbers of visited arms. An analyse of variance revealed that the number of visited arms was greater in $\mathrm{mDS}$ than Sham and IDS-lesioned rats $[(F(1,51)=16.53, p<0.001$ and $F(1,25)=11.25, p<0.005$, respectively], indicating that $\mathrm{mDS}$ rats developed some hyperactivity during the pre-training.
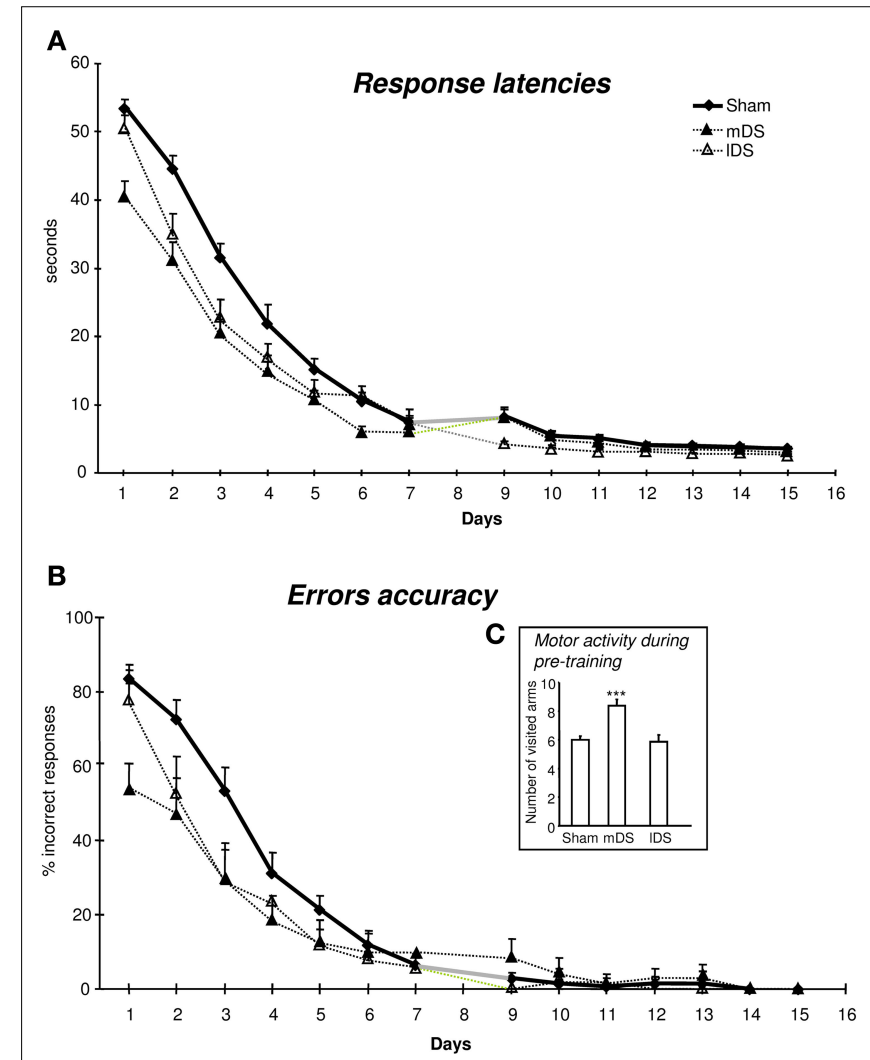

FIGURE 2 | Performance across 15 days of acquisition training in the crossmaze. (A) Group means of response latencies (sec \pm SEM). (B) Group means of percent errors ( \pm SEM) in Sham, mDS and IDS-lesioned rats. (C) Group means of arms visited during pre-training $( \pm$ SEM) in sham, $\mathrm{mDS}$ - and IDS-lesioned rats. 


\section{Acquisition (see Figures 2A,B)}

During the course of training, rats in the different groups displayed a general decrease in their response latencies $[F(13,1170)=307.41$, $p<0.001]$ and in their number of errors $[F(13,1170)=220.23$, $p<0.001]$, with no main effect of lesions $(F$ values $<1)$ nor any interaction between lesion type and extent of training $(F$ values $<1)$. As indicated in Figure 2, mDS rats tended, however to show shorter response latencies and reduced number of errors than Sham rats, confirming the slight hyperactivity already depicted. Figure 2B further indicated Sham and IDS rats exhibited more than $50 \%$ of errors at the beginning of training, due to the fact that some rats failed to make a response within 1 min which was considered as an incorrect response. This has to be related to the relatively long response latencies made by these rats at the beginning of training.

\section{Probe trials (see Figure 3)}

On days 8 and 16, a probe trial was given using a new start arm, opposite to that which rats were trained on. Rats entering the same arm as that during training were designated place learners. Rats entering the opposite arm were designated response learners (Figure 3A).
A

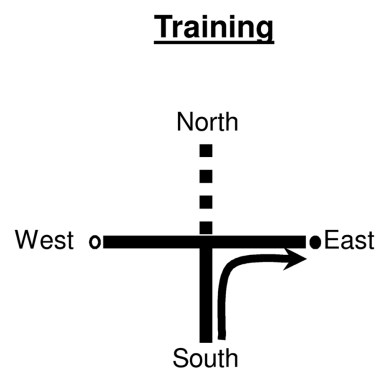

B

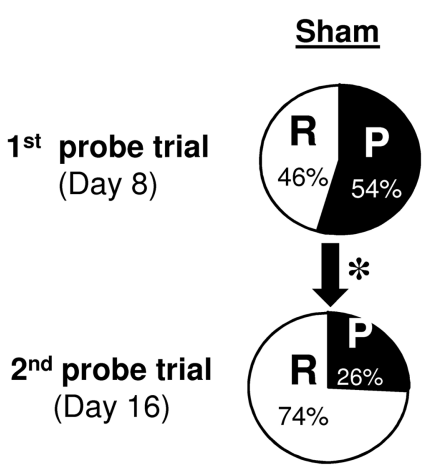

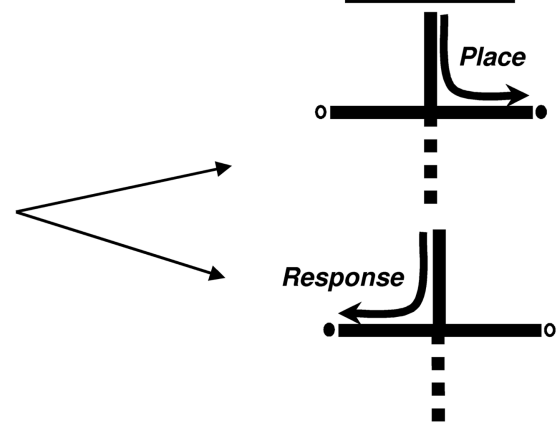

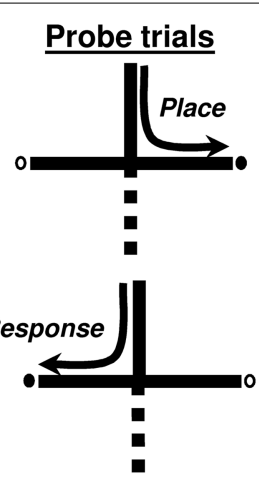

C
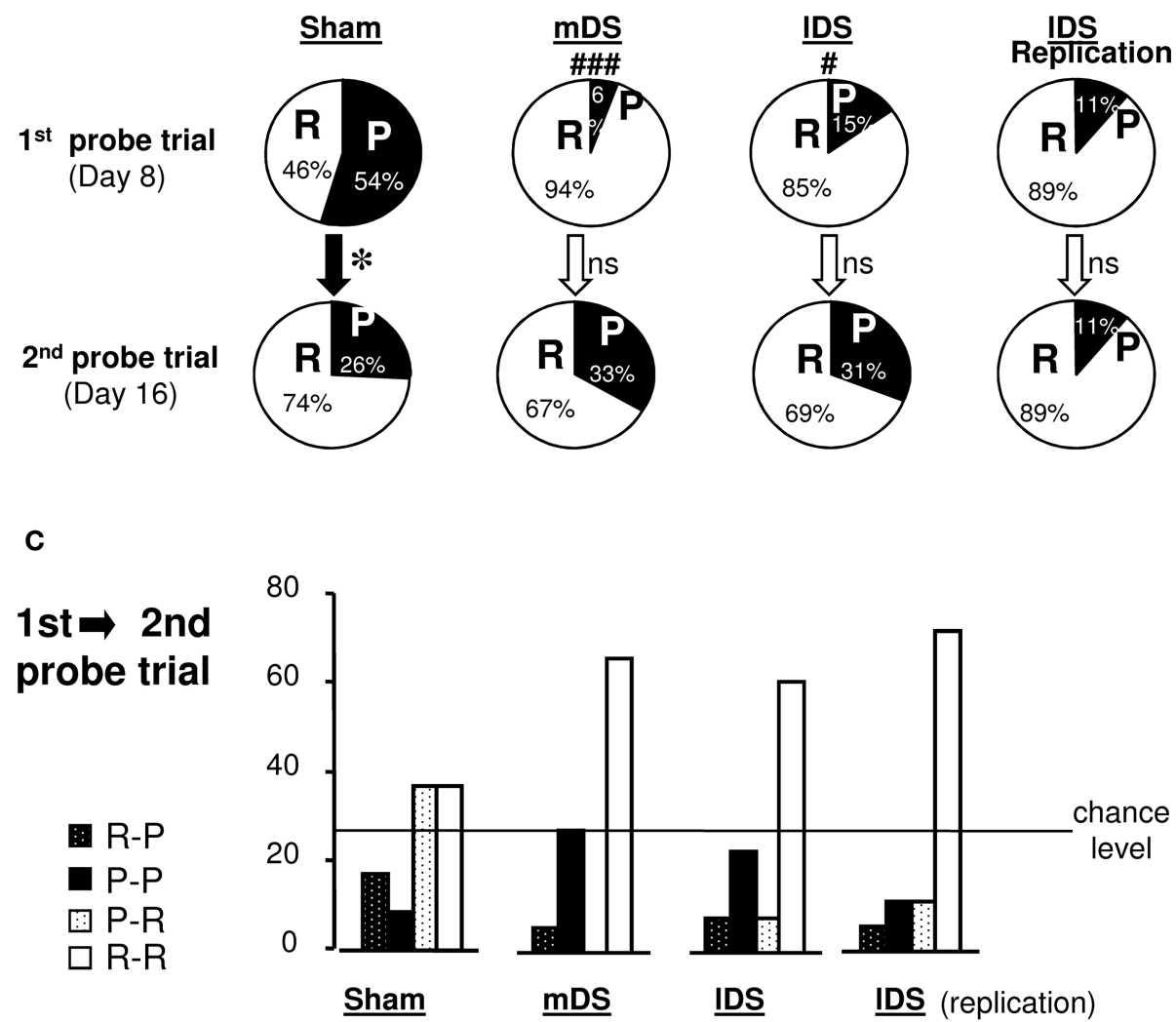

FIGURE 3 | Cross-maze task. (A) Rats were trained starting from the South arm to reach the East (or West) arm of the cross-maze, for four trials a day, from day 1 to 15. During the two probe trials, rats started from the North arm for a single non-reinforced trial. Rats reaching the usual baited arm were termed Place, while those using the same body turn were termed Response. (B) Proportion of Sham,
mDS and IDS lesioned-rats using Place (P) and Response (R) strategies in the cross-maze during the first and the second probe trials. (C) Percentage of rats (Sham and DS lesioned rats) using the same [Place (P-P) or Response (R-R)] strategies, or different strategies (P-R or R-P), during the two probe trials. Between-differences: ${ }^{*} p<0.05$; within-differences: ${ }^{\#} p<0.05$, ${ }^{\# \# \#} p<0.001$. 
On the first probe trial, Sham rats exhibited no preference between place and response strategies. They significantly changed their preferred strategy between day 8 and $16\left(\chi^{2}=5.06, p<0.05\right.$, Figure 3B), and after extended training, they predominantly chose the response strategy $\left(\chi^{2}=8.26, p<0.005\right.$, Figure $\left.2 \mathrm{~A}\right)$, confirming previous results (Packard and McGaugh, 1996). Surprisingly, however, both $\mathrm{mDS}$ and IDS-lesioned rats significantly differed from Sham rats during the first probe trial, exhibiting a strong and significant preference for the response strategy (mDS: $\chi^{2}=17.22, p<0.001$; IDS: $\left.\chi^{2}=5.19, p<0.05\right)$. Both groups maintained a response strategy after extended training, as shown on second probe trial (Figure 3B). Figure 3C illustrated the percentage of rats in each experimental group that express either the same or a different strategy during the second probe trial to that used in the first probe trial. The pattern of response expressed by Sham-rats changed significantly between day 8 and $16\left(\chi^{2}=5.06, p<0.05\right)$ with a stronger tendency to express the response strategy during the second probe trial, regardless of whether this strategy was adopted during the first probe trial. DS lesioned rats mainly used the response strategy during both probe trials. Even though rats exhibited weaker tendencies to use it on day 16 rather than day 8 , their pattern of response was not significantly modified (mDS: $\chi^{2}=3.20, p<0.074$; lDS: $\chi^{2}$ values $<1$ ).

\section{EXPERIMENT 2}

Results from Experiment 1 showing that IDS lesions promote a choice of the response strategy, stand in contrast to the predominant idea that the IDS is involved in stimulus-response associations
(Devan and White, 1999). It was thus decided to replicate the experiment using only rats with excitotoxic lesions of the lateral part of the dorsal striatum $(n=18)$.

\section{Cross-maze task (Figure 3)}

Rats were first trained for 16 days (four trials a day) to reach a constant goal baited arm from a constant start arm, under experimental conditions identical to those used in Experiment 1. Rats showed a general decrease in their response latencies and number of errors (data not shown). As illustrated in Figure 3B, the majority of IDSlesioned rats (15 out of 18), adopted a response strategy during both the first and second probe trial with most of them maintaining the same strategy for the two probe test (see Figure 3C), thereby replicating the previous results.

\section{Complementary experiment on the cross-maze task (Figure 4)}

This complementary experiment was performed on the 15 IDS lesioned rats that exhibited a response strategy on the second probe trial. As these results were unexpected and because the strategy adopted by the rats was inferred on the basis of a single choice performed on the probe trials, we investigated the validity of this assessment in a complementary experiment. Rats were further trained in a modified version of the cross-maze task in which two start arms were now used in alternation (Figure 4A), determining two types of trials: those using the usual south start arm, identical to trials performed in the initial training phase and trials using the north start arm, only experienced during the probe trials. Half of the rats had
A
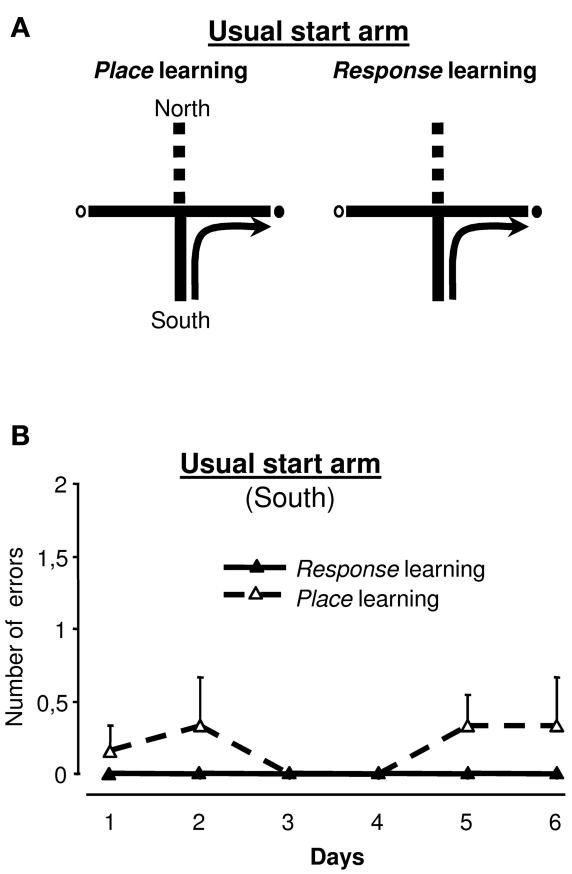

FIGURE 4 | Complementary cross-maze task. (A) IDS-lesioned rats having used a Response strategy during the second probe trial $(n=15)$ were further trained in a modified version of the cross-maze task, using two start arms. Training was identical to the previous experiment when the start arm was the South arm. When rats started from the North arm, half of them were required to reach the same arm (Place learning), while the other half was required to
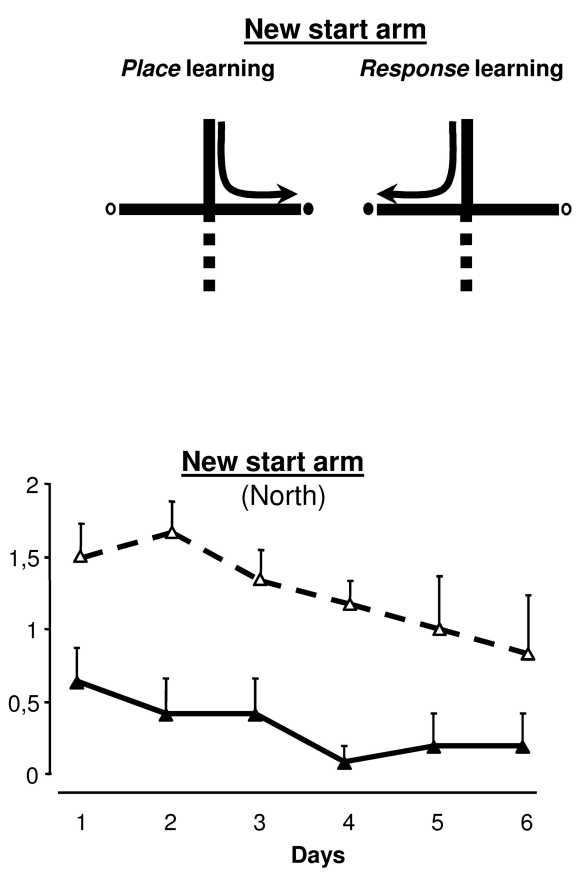

perform the same body turn (Response learning). (B) Group means of number of errors $( \pm$ SEM) made by IDS rats when trained in the modified Response $(n=6)$ or Place $(n=9)$ cross-maze task. Left: There was no between-group difference for the usual start arm (South). Right: Rats acquired the Response significantly faster than the Place task from the new start arm (North). 
to use a place strategy to access the food reinforcer, while the other half had to choose a response strategy. The hypothesis was that if rats were really inclined to use the response strategy (as determined by the probe trials), they would acquire more rapidly response rather than place learning. As illustrated in Figure 4B, when trained from the usual south start arm, the two groups of rats performed similarly, with very few errors. When rats were started from the north start arm, rats trained in a response strategy made significantly fewer errors than those trained with the place strategy $[F(1,13)=25.71, p<0.001]$ indicating that for rats classified as using a response strategy on the basis of the probe test in the cross-maze, this was much easier than the place strategy. This result supports the notion that the probe test used at the end of the cross-maze task correctly identified the preferred strategy and confirmed that IDS lesioned rats favoured a response strategy.

\section{WATER-MAZE TASK}

Damage to the dorsal striatum has been associated with disruption of spatial behaviour (McDonald and White, 1994; Devan and White, 1999; Devan et al., 1999). To examine whether the small proportion of DS-lesioned rats selecting a place strategy could possibly be due to an inability to use spatial cues, rats used in Experiment 1 were further trained in a water maze. At the end of the T-maze task, rats were fed ad libitum and after a 2-week period, they were trained in a water-maze task, using an experimental design that mimicked the test for strategy-choice in the cross-maze.

Rats were trained to find a hidden platform placed in a fixed position, from a constant start position, four trials a day for 15 days (Figure 6A). Strategies were determined on two probe trials (days 8 and 15), using a start point located in the quadrant opposite to that which rats were trained on. This experiment further determines whether the shift of strategies may also occur in another dual-solution task.

\section{Training (see Figure 5)}

Lesioned and Sham groups improved their performance progressively with a significant decrease in response latencies $[F(14,910)=82.78, \quad p<0.001]$ and number of failed trials $[F(14,910)=35.92, p<0.001]$. There was no lesion effect [latencies: $F(4,65)=2.51$, ns; errors: $F(4,65)=1.38$, ns] nor interaction between lesion and day factors [latencies: $F(56,910)=1.2$, ns; errors: $F(56,910)<1]$. mDS-lesioned rats, however, showed longer response latencies and a greater number of errors than Sham [latencies: $F(1,33)=7.55, p<0.01$; errors: $F(1,33)=5$, $p<0.05$ ] and IDS rats [latencies: $F(1,18)=9.93, p<0.01$; errors: $F(1,18)=5.20, p<0.05]$.

\section{A \\ Response latencies}

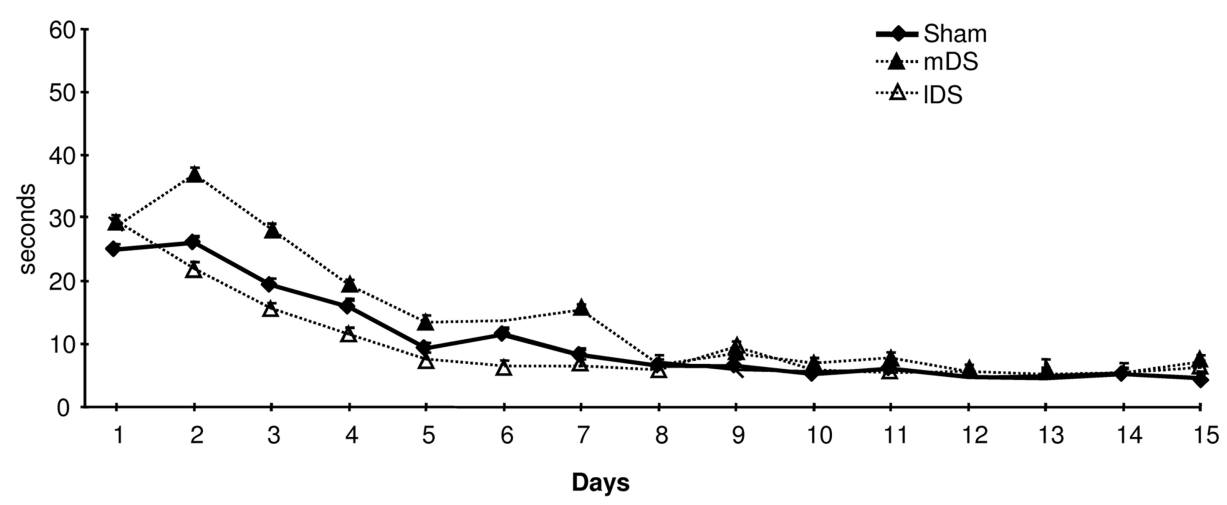

B

Failed trials

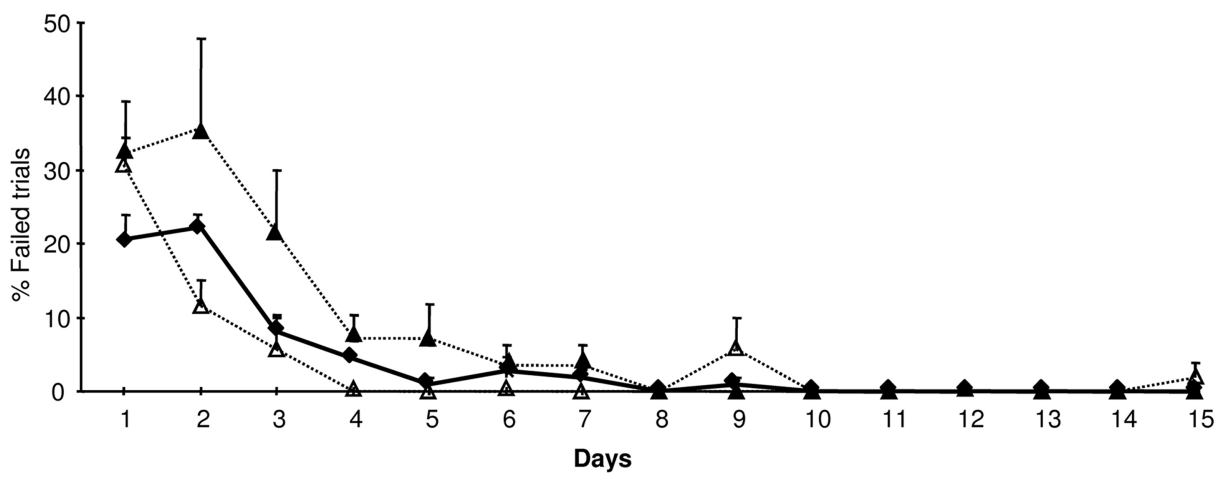

FIGURE 5 | Performance across the 15 training days of acquisition in the water-maze. (A) Group means of response latencies (sec \pm SEM). (B) Group means of failed trials $( \pm S E M)$ in Sham, mDS- and IDS-lesioned rats. 


\section{Probe trials (see Figure 6)}

Rotation of their starting position during a probe trial did not disrupt swimming behaviour and after a short period of uncertainty (lasting just a few second) the animals simply used the available distal cues to search the platform in the vicinity of its original location.
During the 60-s probe test, all rats, regardless of lesion type spent significantly more time in the quadrant in which the platform was located ( $p$ values $<0.001$ ). The same results were observed during the second probe test, indicating that the choice of place strategy was not influenced by the level of training. In addition, the present
A

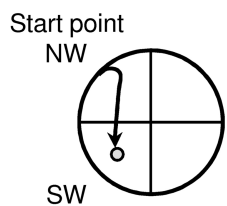

Training

(From Day 1 to Day 15)

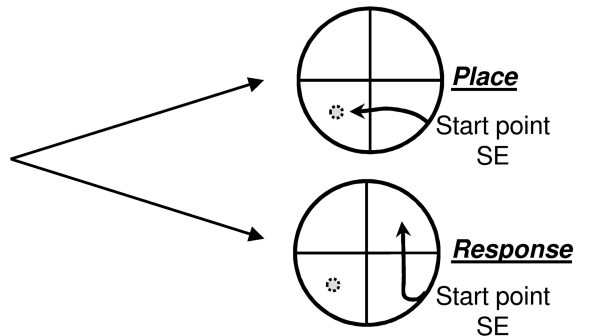

Probe trials

(Day 8 and Day 15)

B

Number of crossings
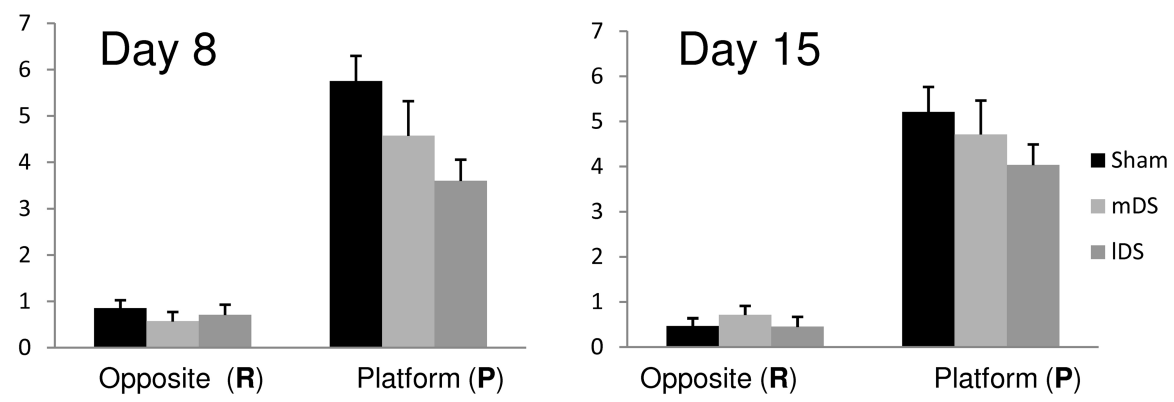

C

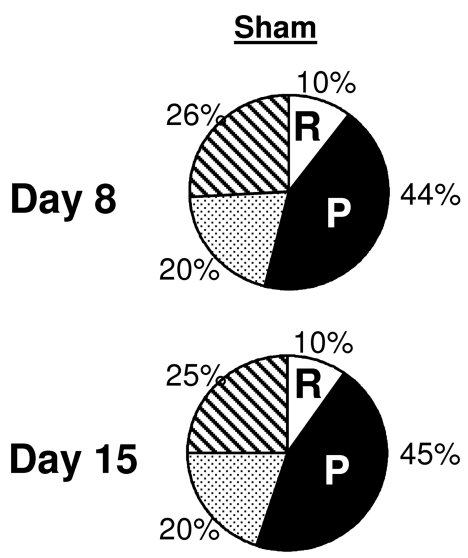

-quadrant of the platform (Place strategy, $\mathbf{P}$ )

-quadrant oposed to the platform (Response strategy, R) p
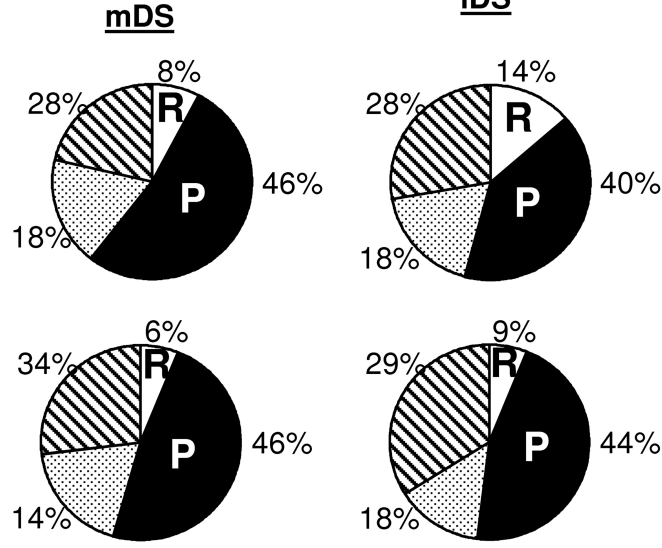

-quadrant of the usual start

$\mathbf{\nabla}$-quadrant of the probe trial start
FIGURE 6 |Water-maze task. (A) Rats were trained from one starting point (NW) to reach one goal location (SW), for four trials a day, from day 1 to 15 . During the 1-min probe trials (at day 8 and 15), rats started from an opposite position (SE) and the time spent in the different quadrants were recorded. (B) Mean number of crossings over the putative platform location (Place strategy, P) and the opposite location (Response strategy, R) in sham, $\mathrm{mDS}$ - and IDS-lesioned rats during the first and second probe trials in the water maze. (C) Proportion of time spent in each quadrant of the water maze in sham and lesioned rats during the two probe trials. Four quadrants have been defined: the quadrant used for start during training, that used for start during the probe trial, that that had containing the platform (P) and the opposite quadrant (R). All rats spent significantly more time in the Place quadrant than in the Response quadrant, irrespectively of the lesion type and the amount of training. 
experiment indicates that neither mDS nor IDS lesions interfered with the ability to use a spatial strategy in a prototypic spatial learning task (see Figure 6B).

\section{DISCUSSION}

The present data indicate that rats trained in a dual-solution crossmaze task tend to shift from a place to a responsestrategy with extended training, confirming previous results (Restle, 1957; Packard and McGaugh, 1996). Unexpectedly, dorsostriatal lesions (IDS and mDS) did not disrupt the ability to use an egocentric response strategy and even increased the preference for this strategy in early training, with no clear dissociation between the two types of lesions. In addition, when the same rats were trained in another dual-solution task performed in a water maze, rats always chose a place strategy, without any strategy shifting across training. Each of these points will be discussed.

\section{THE USE OF PLACE VERSUS RESPONSE STRATEGIES, EARLY IN TRAINING}

Control rats trained in the cross-maze task were almost evenly divided between those displaying place and response strategies. A large number of studies have shown that the use of place and response strategies early in training depends largely on intra- and extra-maze cue arrangements and densities (Tolman et al., 1946, 1947; Restle, 1957; Oliveira et al., 1997; Passino et al., 2002; Canal et al., 2005). The capacity to use environmental cues to drive the behaviour (i.e. the use of a place strategy) seems to be enhanced in an environment full of extra-maze cues. As recently reviewed by Packard (2009), the environment is, however, not the only factor able to modulate the early choice of strategy in a cross-maze task. Distribution of training (Thompson and Thompson, 1949), drugs modulating anxiety (Wingard and Packard, 2008), levels of oestrogen (Zurkovsky et al., 2007), and of cerebral serotonin (Anguiano-Rodríguez et al., 2007) all exert an influence on the type of strategy adopted early during training in the cross-maze, accounting for the difference in results frequently observed during the earlier test.

\section{RESPONSE STRATEGIES AND EXTENDED TRAINING}

It seems now rather well established that rats strongly favour response strategy after extended training in a dual-solution task tested on in a cross-maze (Ritchie et al., 1950; Restle, 1957; Hicks, 1964; Mitchell and Hall, 1988; Packard and McGaugh, 1996; Oliveira et al., 1997; Passino et al., 2002; Yin and Knowlton, 2004). The present results showing that $74 \%$ of the control rats exhibited a response strategy during the second probe trial, confirmed these previous findings.

In this experiment, rats were further trained in another dualsolution task tested in a water-maze, that involved a fixed start and goal position. Under these conditions rats always maintained place strategy, even following extended training (60 trials). There is some evidence indicating that rats can use egocentric/response strategies to guide their behaviour in the water maze, but in these conditions, the acquisition is rather slow (de Bruin et al., 1997, 2001). Even if we cannot exclude the possibility that a shift towards automatic motor response, could take place after additional training the present results show that such responses are not preferentially implemented (see also McGauran et al., 2004; Kealy et al., 2008 for similar results). This may be due to differences in the potential to automate a running response into a particular arm, and a swimming response in an open space like a water maze.

\section{EFFECTS OF IDS AND mDS LESIONS IN THE STRATEGY CHOICE}

The present study does not show any dissociation of function between the IDS and mDS in a dual-solution cross-maze task, a result which has previously been suggested (Pisa and Cyr, 1990). In our experiments, both types of lesions: either the lateral or medial part of the dorsal striatum induced an increase in choice of the response strategy during the first probe test that occurred after seven training days (i.e. after 28 training trials). Contrary to control rats which significantly increase their use of the response strategy as training progressed, DS lesioned rats exhibited a slight tendency to decrease its use, possibly suggesting that rats did not properly implement the motor habit.

\section{Effects of $m D S$ lesions in the strategy choice}

The present result indicate that excitotoxic lesions to the $\mathrm{mDS}$ prior to training leads to greater reliance on a response strategy in lesioned rats compared with controls when tested after limited training. A similar result has already been shown in a previous study with lesions in the most posterior part of the mDS; a region considered to be a part of a corticostriatal system involved in the processing of the spatial information (Yin and Knowlton, 2004). As the present lesions are not so restricted, it may be that the whole $\mathrm{mDS}$ which has functional connections with the hippocampus, could be part of a system mediating flexible, goal-directed responding, based on an integrated representation of the environment (Devan et al., 1999; Yin and Knowlton, 2004). Experiment 2, however, indicated that, if any, the role of mDS in the spatial memory system is rather weak, as our rats with $\mathrm{mDS}$ lesions remained capable of using spatial strategies during a probe test in a water-maze task, even when trained to reach a fixed position from a fixed starting point (see also Devan and White, 1999). Alternatively, it has been suggested that the $\mathrm{mDS}$ lesions alter sensory information processing (Pisa and Cyr, 1990) and more particularly the processing of visual information (Kamishina et al., 2008). The lack of difference between lesioned and control rats indicates that if any alteration in spatial and visual information occurred, they were compensated for. This might happen, for example, by the use of vestibular signals which have been shown to promote accurate water maze performance in hippocampal deficient mice (Middei et al., 2007). Rats with $\mathrm{mDS}$ lesions might be viewed as tending to focus on the lower-cost, task specific, strategy in a given situation, i.e. the response strategy in our cross-maze task and the place strategy in the water-maze task and to lose some behavioural flexibility such as the one subserving strategy shifting (Ragozzino, 2007). Accordingly, a relatively subtle deficit might only be detected in specific conditions, such as when rats are left to freely choose between several available strategies, as in the present conditions. This would account for the fact that when rats are forced to adopt a particular strategy, such as in a discriminative task, neither permanent nor transitory mDS lesions seem to induce any disruption, which could well account for previously published results (Pisa and Cyr, 1990; Ragozzino et al., 2002).

\section{Effects of IDS lesions in the strategy choice}

The most unexpected results in the present study were those concerning rats with IDS lesions. While IDS lesions have been shown to prevent the use of the response strategy (Potegal, 1972; McDonald and White, 1993; Devan et al., 1999), we find that, in fact, the lesion favours its use from the early phase of training. The results shown in our complementary experiment indicate that IDS lesioned rats, 
using a response strategy during the second probe test, were much quicker at learning a response than a place task, demonstrating that the probe test provided a valid appraisal of the strategy used by the rats in solving the cross-maze task. We further showed that lesioned rats, as controls, adopted a place strategy when trained in the water maze, indicating that the increased use of the response strategy by rats with IDS lesions was not due to difficulties in using spatial information.

That IDS lesioned rats used the response strategy significantly more than the control rats does not demonstrate a lack of shift from the place to the response strategy in these rats. Although rather unlikely, it is possible that IDS lesions accelerate learning processes in such a way that the shift occurred before the first probe trial. The key point in this study, however, is not to determine whether a strategy shift occurred or not, but to show that most of the rats with IDS lesions were able to use the response strategy, while IDS is generally considered as the neural substrate for response strategy (Potegal, 1972; McDonald and White, 1993; Devan et al., 1999).

Surprisingly, such an increased use of the response strategy early in training in rats with IDS dysfunctions is not unique in the literature. Pre-training IDS injections of artificial cerebral spinal fluid (Canal et al., 2005), and post-training injections of glutamate into the IDS (presumed to induce striatal activation; Packard, 1999) have been shown to increase the use of the response strategy in very similar procedures. Such an effect could thus be interpreted as resulting from a IDS dysfunction due to intra striatal injections. It is, however, important to note that IDS injections did not always increase the use of the response strategy, and no alteration of the early pattern of strategies have been reported with pre-training IDS injections of glucose (Canal et al., 2005) and with pretest injection of lidocaine (Packard and McGaugh, 1996), which is considered to induce transient lesions (but see Gisquet-Verrier and Delatour, 2006 for a dissociation between lesion and transient inactivation).

Our results using pre-training $\mathrm{IDS}$ lesions contrast with the results of a previous study (Yin and Knowlton, 2004), which showed that IDS-lesioned rats predominantly chose a place strategy during both probe tests, with a cross-maze surrounded by a curtain providing no extra-maze cues, except the light of an opened door. Under these conditions, it is difficult to imagine how a place strategy can be used. One may rather suggest that in this situation, offering a choice between a response and a cuestrategy, rats with IDS lesions favour a cue strategy, while in our conditions offering choice between a response and a place strategy, rats with IDS lesions favour a response strategy.

Accordingly, all these data appear to be compatible with the idea that IDS lesions do not affect the rats' abilities to use a particular type of strategy (place, response and cue). The IDS has frequently been described as an important structure for the identification or selection of relevant information which will allow associations of particular sets of cues with reinforcement, i.e. to select a response (Reading et al., 1991; Brasted et al., 1999; Featherstone and McDonald, 2004). This is well illustrated by a study performed by Chang and Gold (2004) who trained rats in a response task in a cross-maze surrounded or not by environmental cues. They showed that rats with IDS inactivation performed similarly in the two situations, while control rats were quicker to adopt the response strategy in the absence of environmental cues (i.e. in a situation where the response strategy was the only available). They concluded that IDS inactivation disrupted egocentric discrimination only in a non cue condition. We may alternatively propose that rats with IDS inactivation showed poorer processing of the information relative to the surrounding environment of the cross-maze, due to a disruption in selective attention and/or visual detection processes.

\section{CONCLUSION}

Although the precise specification of role of IDS and $\mathrm{mDS}$ requires complementary experiments, the present experiments demonstrate that in our particular conditions, rats with $m$ and IDS lesions were able to select and use the response strategy normally, and even more than control animals. Reconsidering the literature in light of our results, it appears that the dorsal striatum may not be required when rats have to adopt a particular strategy or when a strategy is predictable such as the place strategy in the water maze. On the other hand, lesions to the dorsal striatum, may modify the choice of the strategy when rats are free to choose between several similarly effective strategies. The alteration of attention and/or sensory processing, may strengthen response, place, or cue strategies, depending on the surrounding environment. Such an explanation could account for the contradictory results in the literature concerning the role of dorsal striatum in egocentric discriminations (Potegal, 1969; Cook and Kesner, 1988; Kesner et al., 1993; DeCoteau and Kesner, 2000 versus Divac et al., 1967, 1978; Kirkby, 1969; Pisa and Cyr, 1990; Oliveira et al., 1997).

In our conditions, DS lesions increased the choice of the response strategy during early training with no clear difference between $\mathrm{mDS}$ and IDS lesions. The early use of a response strategy in DS lesioned rats is probably not due to more rapidly acquired habit formation, but could reveal inability to take into account the environmental cues, leading them to behave as if they were in a no cue condition, as already proposed by Chang and Gold (2004). Recent evidence indicates that the central part of the SD receives inputs from several cortical areas including the medial agranular cortex, posterior parietal cortex and visual association cortex. Together, these regions form a cortical-subcortical circuit involved in directed attention and neglect (Kamishina et al., 2008). The fact that these different inputs converge at the central part of the SD could account for the similarity of results obtained in the present study with lesions in the lateral and the medial part of the SD.

These results, which have been confirmed with an additional and a complementary experiment, indicate that the IDS cannot be considered as a neural support for the response memory system. They further indicate that alteration of the strategy used to solve a task is not systematically due to inability to adopt place or response strategies, but may reveal more subtle deficits concerning the processing of sensory information, and/or cue selection which is involved in the strategy choices. It may be time to reconsider the role of the medial and lateral part of the dorsal striatum in egocentric/response memory, which seems to play a more refined role in mediating this kind of learning than was initially proposed.

\section{ACKNOWLEDGMENTS}

The authors are grateful to Nathalie Samson and Pascale Veyrac for animal care. Our deepest thanks go to Sabrina Davis for her work on the English text. This work was supported by CNRS, and by grants from the French Ministry of Research (ACI-NEURO-NIC042133) to Pascale Gisquet-Verrier; Fanny Botreau was supported by a fellowship from the French Ministry of Research. 


\section{REFERENCES}

Anguiano-Rodríguez,P.B.,Gaytán-Tocavén, L., and Olvera-Cortés, M. E. (2007). Striatal serotonin depletion facilitates rat egocentric learning via dopamine modulation. Eur. J. Pharmacol. 556, 91-98.

Barth, T. M., Jones, T. A., and Schallert, T. (1990). Functional subdivisions of the rat somatic sensorimotor cortex. Behav. Brain Res. 39, 73-95.

Brasted, P. J., Robbins, T.W., and Dunnett, S. B. (1999). Distinct roles for striatal subregions in mediating response processing revealed by focal excitotoxic lesions. Behav. Neurosci. 113, 253-264.

Canal, C. E., Stutz, S. J., and Gold, P. E. (2005). Glucose injections into the dorsal hippocampus or dorsolateral striatum of rats prior to T-maze training: modulation of learning rates and strategy selection. Learn. Mem. 12, 367-374.

Castro-Alamancos, M. A., and Borrel, J. (1995). Functional recovery of forelimb response capacity after forelimb primary motor cortex damage in the rat is due to the reorganization of adjacent areas of cortex. Neuroscience 68, 793-805.

Chang, Q., and Gold, P. E. (2004). Inactivation of dorsolateral striatum impairs acquisition of response learning in cue-deficient, but not cue-available, conditions. Behav. Neurosci. 118, 383-388.

Cook, D., and Kesner, R. P. (1988). Caudate nucleus and memory for egocentric localization. Behav. Neural Biol. 49, 332-343.

de Bruin, J. P., Moita, M. P., de Brabander, H. M., and Joosten, R. N. (2001). Place and response learning of rats in a Morris water maze: differential effects of fimbria fornix and medial prefrontal cortex lesions. Neurobiol. Learn. Mem. 75, 164-178.

de Bruin, J. P., Swinkels, W. A., and de Brabander, J. M. (1997). Response learning of rats in a Morris water maze: involvement of the medical prefrontal cortex. Behav. Brain Res. $85,47-55$.

DeCoteau, W. E., and Kesner, R. P. (2000). A double dissociation between the rat hippocampus and medial caudoputamen in processing two forms of knowledge. Behav. Neurosci. 114, 1096-1108.

Delatour, B., and Gisquet-Verrier, P. (1996). Prelimbic cortex specific lesions disrupt delayed-variable response tasks in the rat. Behav. Neurosci. 110, 1282-1298.

Devan, B. D., McDonald, R. J., and White, N. M. (1999). Effects of medial and lateral caudate-putamen lesions on place- and cue- guided behaviors in the water maze: relation to thigmotaxis. Behav. Brain Res. 100, 5-14.

Devan, B. D., and White, N. M. (1999). Parallel information processing in the dorsal striatum: relation to hippocampal function. J. Neurosci. 19, 2789-2798.

Divac, I. (1968). Functions of the caudate nucleus. Acta Neurobiol. Exp. (Wars) 28, 107-120.

Divac, I., and Diemer, N. H. (1980) Prefrontal system in the rat visualized by means of labeled deoxyglucose - further evidence for functional heterogeneity of the neostriatum. J. Comp. Neurol. 190, 1-13.

Divac, I., Markowitsch, H. J., and Pritzel, M. (1978). Behavioral and anatomical consequences of small intrastriatal injections of kainic acid in the rat. Brain Res. 151, 523-532.

Divac, I., Rosvold, H.E., and Szwarcbart, M K. (1967). Behavioral effects of selective ablation of the caudate nucleus. J. Comp. Physiol. Psychol. 63, 184-190.

Donoghue, J. P., and Herkenham, M. (1986). Neostriatal projections from individual cortical fields conform to histochemically distinct striatal compartments in the rat. Brain Res. 365, 397-403.

Dunnett, S. B. (1990). Role of prefrontal cortex and striatal output systems in short-term memory deficits associated with ageing, basal forebrain lesions, and cholinergic-rich grafts. Can. J. Psychol. 44, 210-232.

Eichenbaum, H. (2001). The hippocampus and declarative memory: cognitive mechanisms and neural codes. Behav. Brain Res. 127, 199-207.

Featherstone, R. E., and McDonald, R. J. (2004). Dorsal striatum and stimulus-response learning: lesions of the dorsolateral, but not dorsomedial, striatum impair acquisition of a stimulus-response-based instrumental discrimination task, while sparing conditioned place preference learning. Neuroscience 124, 23-31.

Gisquet-Verrier, P., and Delatour, B. (2006). The role of the rat prelimbic/ infralimbic cortex in working memory: not involved in the short-term maintenance but in monitoring and processing functions. Neuroscience 141, 585-596.

Hicks, L. H. (1964). Effects of overtraining on acquisition and reversal of place and response learning. Psychol. Rep. 15 459-462.

Kamishina, H., Yurcisin, G. H., Corwin, J V., and Reep, R. L. (2008). Striatal projections from the lateral posterior thalamic nucleus. Brain Res. 1204, 24-39.

Kealy, J., Diviney, M., Kehoe, E. McGonagle, V., O'Shea, A., Harvey, D. and Commins, S. (2008). The effects of overtraining in the Morris water maze on allocentric and egocentric learning strategies in rats. Behav. Brain Res. 192 259-263.

Kesner, R. P., Bolland, B. L., and Dakis, M. (1993). Memory for spatial locations, motor responses, and objects: triple dissociation among the hippocampus, caudate nucleus, and extrastriate visual cortex. Exp. Brain Res. 93, 462-470.

Kesner, R. P., Hunt, M. E., Williams, J. M. and Long, J. M. (1996). Prefrontal cortex and working memory for spatial response, spatial location, and visual object information in the rat. Cereb. Cortex 6, 311-318.

Kirkby, R. J. (1969). Caudate nucleus lesions impair spontaneous alternation. Percept. Mot. Skills 29, 550.

Kolb, B. (1977). Studies on the caudateputamen and the dorsomedial thalamic nucleus of the rat: implications for mammalian frontal-lobe functions. Physiol. Behav. 18, 237-244.

Krettek, J. E., and Price, J. L. (1977). The cortical projections of the mediodorsal nucleus and adjacent thalamic nuclei in the rat. J. Comp. Neurol. 171, 157-191.

McCool, M. F., Patel, S., Talati, R., and Ragozzino, M. E. (2008). Differentia involvement muscarinic cholinergic receptors in the dorsomedial striatum in task switching. Neurobiol. Learn. Mem. 89, 114-124.

McDonald, R. J., and White, N.M. (1993).A triple dissociation of memory systems: hippocampus, amygdala, and dorsa striatum. Behav. Neurosci. 107, 3-22.

McDonald, R. J., and White, N. M. (1994) Parallel information processing in the water maze: evidence for independent memory systems involving dorsal striatum and hippocampus. Behav. Neural Biol. 61, 260-270.

McGauran,A.M.,Harvey,D.,Cunningham, L., Craig, S., and Commins, S. (2004) Retention of cue-based associations in the water maze is time-dependent and sensitive to disruption by rotating the starting position. Behav. Brain Res. 151, 255-266.

Middei, S., Vetere, G., Sgobio, C., and Ammassari-Teule, M. (2007). Landmark-based but not vestibular-based orientation elicits mossy fiber synaptogenesis in the mouse hippocampus. Neurobiol.Learn. Mem 87, 174-180.

Mishkin, M., and Petri, H. L. (1984) Memories and Habits: Some Implications for the Analysis of Learning and Retention. New York, Guilford.

Mitchell, J. A., and Hall, G. (1988) Caudate-putamen lesions in the rat may impair or potentiate maze learning depending upon availability of stimulus cues and relevance of response cues. Q. J. Exp. Psychol. B 40,243-258.

Oliveira, M. G., Bueno, O. F., Pomarico, A. C., and Gugliano, E. B. (1997). Strategies used by hippocampal- and caudate-putamen-lesioned rats in a learning task. Neurobiol. Learn. Mem. $68,32-41$.

Packard, M.G. (1999). Glutamate infused posttraining into the hippocampus or caudate-putamen differentially strengthens place and response learning. Proc. Natl. Acad. Sci. U.S.A. 96, 12881-12886.

Packard, M. G. (2009). Exhumed from thought: basal ganglia and response learning in the plus-maze. Behav. Brain Res. 199, 24-31.

Packard, M. G., Hirsh, R., and White, N. M. (1989). Differential effects of fornix and caudate nucleus lesions on two radial maze tasks: evidence for multiple memory systems. J. Neurosci. 9 , 1465-1472.

Packard, M. G., and McGaugh, J. L. (1996). Inactivation of hippocampus or caudate nucleus with lidocaine differentially affects expression of place and response learning. Neurobiol. Learn. Mem. 65, 65-72.

Palencia, C. A., and Ragozzino, M. E. (2004). The influence of NMDA receptors in the dorsomedial striatum on response reversal learning. Neurobiol. Learn. Mem. 82, 81-89.

Passino, E., Middei, S., Restivo, L., BertainaAnglade, V., and Ammassari-Teule, $\mathrm{M}$. (2002). Genetic approach to variability of memory systems: analysis of place vs. response learning and fos-related expression in hippocampal and striatal areas of C57BL/6 and DBA/2 mice. Hippocampus 12, 63-75.

Pisa, M., and Cyr, J. (1990). Regionally selective roles of the rat's striatum in modality-specific discrimination learning and forelimb reaching. Behav. Brain Res. 37, 281-292.

Potegal, M. (1969). Role of the caudate nucleus in spatial orientation of rats. J. Comp. Physiol. Psychol. 69, 756-764.

Potegal, M. (1972). The caudate nucleus egocentric localization system. Acto Neurobiol. Exp. (Wars) 32, 479-494.

Ragozzino, M. E. (2007). The contribution of the medial prefrontal cortex, orbitofrontal cortex, and dorsomedial striatum to behavioral flexibility. Ann. N. Y. Acad. Sci. 1121, 355-375.

Ragozzino, M. E., Jih, J., and Tzavos, A (2002). Involvement of the dorsomedial striatum in behavioral flexibility: role of muscarinic cholinergic receptors. Brain Res. 953, 205-214. 
Reading, P. J., Dunnett, S. B., and Robbins, T. W. (1991). Dissociable roles of the ventral, medial and lateral striatum on the acquisition and performance of a complex visual stimulus-response habit. Behav. Brain Res. $45,147-161$.

Restle, F. (1957).Discrimination of cues in mazes: a resolution of the place versus response controversy. Psychol. Rev. 64, 217-228.

Ritchie, B. F., Aeschliman, B., and Pierce, P. (1950). Studies in spatial learning: VIII. Place performance and acquisition of place dispositions. J. Comp. Physiol. Psychol. 43, 73-85.

Rouanet, H., Bernard, J. M., and Leroux, B. (1990). Statistiques en sciences humaines: Analyse inductive des données. Paris, Dunod.

Sesack, S. R., Deutch, A.Y., Roth, R. H., and Bunney, B. S. (1989). Topographical organization of the efferent projections of the medial prefrontal cortex in the rat: an anterograde tract-tracing study with Phaseolus vulgaris leucoagglutinin. J. Comp. Neurol. 290, 213-242.
Thompson, M. E., and Thompson, J. P. (1949). Reactive inhibition as a factor in maze learning: II. The role of reactive inhibition in studies of place learning versus response learning. $J$. Exp. Psychol. 39, 883-891.

Thompson, W. G., Guilford, M. O., and Hicks, L. H. (1980). Effects of caudate and cortical lesions on place and response learning in rats. Physiol. Psychol. 8, 473-479.

Tolman, E. C., Ritchie, B. F., and Kalish, D. (1946). Studies in spatial learning: II. Place learning versus response learning. J. Exp. Psychol. 35, 221-229.

Tolman, E. C., Ritchie, B. F., and Kalish, D. (1947). Studies in spatial learning: V. Response versus place learning by the noncorrection method. J. Exp. Psychol. 37, 285-292.

Tzavos, A., Jih, J., and Ragozzino, M. E. (2004). Differential effects of M1 muscarinic receptor blockade and nicotinic receptor blockade in the dorsomedial striatum on response reversal learning. Behav. Brain Res. 154, 245-253.
Van Golf Racht-Delatour, B., and El Massioui, N. (1999). Rule-based learning impairment in rats with lesions to the dorsal striatum. Neurobiol. Learn. Mem. 72, 47-61.

Whishaw, I. Q., Mittleman, G., Bunch, S. T., and Dunnett,S.B.(1987). Impairments in the acquisition, retention and selection of spatial navigation strategies after medial caudate-putamen lesions in rats. Behav. Brain Res. 24, 125-138.

Wingard, J. C., and Packard, M. P. (2008). The amygdala and emotional modulation of competition between cognitive and habit memory. Behav. Brain Res. 193, 126-131.

Wise, S. P., and Jones, E. G. (1977). Cells of origin and terminal distribution of descending projections of the rat somatic sensory cortex. J. Comp. Neurol. 175, 129-157.

Yin, H. H., and Knowlton, B. J. (2004). Contributions of striatal subregions to place and response learning. Learn. Mem. 11, 459-463.

Zurkovsky, L., Brown, S. L., Boyd, S. E., Fell, J. A., and Korol, D. L. (2007). Estrogen modulates learning in female rats by acting directly at distinct memory systems. Neuroscience 144, 26-37.

Conflict of Interest Statement: The authors declare that the research was conducted in the absence of any commercial or financial relationships that could be construed as a potential conflict of interest.

Received: 03 December 2009; paper pending published: 03 January 2010; accepted: 08 February 2010; published online: 26 February 2010.

Citation: Botreau F and Gisquet-Verrier $P$ (2010) Re-thinking the role of the dorsal striatum in egocentric/response strategy. Front. Behav. Neurosci. 4:7. doi: 10.3389/neuro.08.007.2010

Copyright (C) 2010 Botreau and GisquetVerrier. This is an open-access article subject to an exclusive license agreement between the authors and the Frontiers Research Foundation, which permits unrestricted use, distribution, and reproduction in any medium, provided the original authors and source are credited. 